\title{
Existence and Ulam Stability of Solutions for Discrete Fractional Boundary Value Problem
}

\author{
Fulai Chen ${ }^{1}$ and Yong Zhou ${ }^{2}$ \\ ${ }^{1}$ Department of Mathematics, Xiangnan University, Chenzhou 423000, China \\ ${ }^{2}$ School of Mathematics and Computational Science, Xiangtan University, Xiangtan 411005, China \\ Correspondence should be addressed to Fulai Chen; cflmath@163.com
}

Received 28 May 2013; Accepted 9 July 2013

Academic Editor: Shurong Sun

Copyright (c) 2013 F. Chen and Y. Zhou. This is an open access article distributed under the Creative Commons Attribution License, which permits unrestricted use, distribution, and reproduction in any medium, provided the original work is properly cited.

We discuss the existence of solutions for antiperiodic boundary value problem and the Ulam stability for nonlinear fractional difference equations. Two examples are also provided to illustrate our main results.

\section{Introduction}

This paper investigates the existence of solutions for antiperiodic boundary value problem and the Ulam stability for nonlinear fractional difference equations:

$$
\begin{array}{r}
\Delta_{C}^{\alpha} x(t)=f(t+\alpha-1, x(t+\alpha-1)), \\
t \in[0, b]_{\mathbb{N}_{0}}, \quad 1<\alpha<2,
\end{array}
$$

where $\Delta_{C}^{\alpha}$ is a Caputo fractional difference operator, $\mathbb{N}_{a}=$ $\{a, a+1, a+2, \ldots\}$ and $I_{\mathbb{N}_{a}}=I \bigcap \mathbb{N}_{a}$ for any number $a \in R$ and each interval $I$ of $R, b \in \mathbb{N}_{1}$, and $f:[\alpha-1, b+\alpha]_{\mathbb{N}_{\alpha-1}} \times R \rightarrow R$ is a continuous function with respect to the second variable.

Accompanied with the development of the theory on fractional differential equations, fractional difference equations have also been studied more intensively of late. In particular, some properties and inequalities of the fractional difference calculus are discussed in [1-7], the existence and asymptotic stability of the solutions for fractional difference equations are investigated in [8-10], and the boundary value problems of fractional difference equations are considered in [11-13]. But there are a lot of works to do in the future, and to the best of my knowledge, there is no work on the existence of solutions for antiperiodic boundary value problem and the Ulam stability for nonlinear fractional difference equations.

To research the boundary value problem of fractional difference equations, we need to select a suitable fixed-point theorem because of the discrete property of the difference operator; here, we choose the Banach contraction mapping principle and the Brower fixed-point theorem. Motivated by the work of the Ulam stability for fractional differential equations [14], in this paper, we also introduce four types of the Ulam stability definitions for fractional difference equations and study the Ulam-Hyers stable and the UlamHyers-Rassias stable.

The rest of the paper is organized as follows. In Section 2, we introduce some useful preliminaries. In Section 3, we consider the existence of solutions for antiperiodic boundary value problem of fractional difference equations. In Section 4, we discuss the Ulam stability for fractional difference equations. Finally, two examples are given to illustrate our main results.

\section{Preliminaries}

In this section, we introduce preliminary facts which are used throughout this paper.

Definition 1 (see $[3,4]$ ). Let $v>0$. The $v$ th fractional sum of $f: \mathbb{N}_{a} \rightarrow R$ is defined by

$$
\Delta^{-v} f(t)=\frac{1}{\Gamma(\nu)} \sum_{s=a}^{t-\nu}(t-s-1)^{(\nu-1)} f(s), \quad t \in \mathbb{N}_{a+\nu}
$$

where $t^{(v)}=\Gamma(t+1) / \Gamma(t-v+1)$. 
In (2), the fractional sum $\Delta^{-v}$ maps functions defined on $\mathbb{N}_{a}$ to functions defined on $\mathbb{N}_{a+v}$. Atici and Eloe [3] pointed out that this definition is the development of the theory of the fractional calculus on time scales.

Definition 2 (see [1]). Let $\mu>0$ and $n-1<\mu<n$, where $n$ denotes a positive integer and $n=\lceil\mu\rceil, \Gamma \cdot\rceil$ ceiling of number. Set $\nu=n-\mu$. The $\mu$ th fractional Caputo difference operator is defined as

$$
\begin{aligned}
\Delta_{C}^{\mu} f(t) & =\Delta^{-v}\left(\Delta^{n} f(t)\right) \\
& =\frac{1}{\Gamma(\nu)} \sum_{s=a}^{t-v}(t-s-1)^{(\nu-1)} \Delta^{n} f(s), \quad \forall t \in N_{a+v},
\end{aligned}
$$

where $\Delta^{n}$ is the $n$th order forward difference operator; the fractional Caputo like difference $\Delta_{C}^{\mu}$ maps functions defined on $\mathbb{N}_{a}$ to functions defined on $\mathbb{N}_{a-\mu}$.

Lemma 3 (see $[2,13])$. Assume that $\mu>0$ and $f$ is defined on $\mathbb{N}_{a}$. Then,

$$
\begin{aligned}
\Delta^{-\mu} \Delta_{C}^{\mu} f(t) & =f(t)-\sum_{k=0}^{n-1} \frac{(t-a)^{(k)}}{k !} \Delta^{k} f(a) \\
& =f(t)+c_{0}+c_{1} t+\cdots+c_{n-1} t^{(n-1)},
\end{aligned}
$$

where $n$ is the smallest integer greater than or equal to $\mu, c_{i} \in$ $R, i=1,2, \ldots, n-1$.

Lemma 4. One has

$$
\sum_{s=0}^{t-\alpha}(t-s-1)^{(\alpha-1)}=\frac{\Gamma(t+1)}{\alpha \Gamma(t-\alpha+1)} .
$$

Proof. For $x>k, x, k \in R, k>-1, x>-1$, we have [1]

$$
\begin{aligned}
& \frac{\Gamma(x+1)}{\Gamma(k+1) \Gamma(x-k+1)} \\
& \quad=\frac{\Gamma(x+2)}{\Gamma(k+2) \Gamma(x-k+1)}-\frac{\Gamma(x+1)}{\Gamma(k+2) \Gamma(x-k)} ;
\end{aligned}
$$

that is,

$$
\frac{\Gamma(x+1)}{\Gamma(x-k+1)}=\frac{1}{k+1}\left[\frac{\Gamma(x+2)}{\Gamma(x-k+1)}-\frac{\Gamma(x+1)}{\Gamma(x-k)}\right] .
$$

Then,

$$
\begin{aligned}
\sum_{s=0}^{t-\alpha}(t- & s-1)^{(\alpha-1)} \\
= & \frac{\Gamma(t)}{\Gamma(t-\alpha+1)}+\frac{\Gamma(t-1)}{\Gamma(t-\alpha)}+\cdots+\frac{\Gamma(\alpha+1)}{\Gamma(2)}+\Gamma(\alpha) \\
= & \frac{1}{\alpha}\left(\frac{\Gamma(t+1)}{\Gamma(t-\alpha+1)}-\frac{\Gamma(t)}{\Gamma(t-\alpha)}\right) \\
& +\frac{1}{\alpha}\left(\frac{\Gamma(t)}{\Gamma(t-\alpha)}-\frac{\Gamma(t-1)}{\Gamma(t-\alpha-1)}\right) \\
& +\cdots+\frac{1}{\alpha}\left(\frac{\Gamma(\alpha+2)}{\Gamma(2)}-\frac{\Gamma(\alpha+1)}{\Gamma(1)}\right)+\Gamma(\alpha) \\
= & \frac{\Gamma(t+1)}{\alpha \Gamma(t-\alpha+1)} .
\end{aligned}
$$

The following result is an immediate consequence of Lemma 4.

Corollary 5. One has

(i) $\sum_{s=0}^{b}(b+\alpha-s-1)^{(\alpha-1)}=\Gamma(b+\alpha+1) / \alpha \Gamma(b+1)$,

(ii) $\sum_{s=0}^{b-1}(b+\alpha-s-2)^{(\alpha-2)}=(1 / \alpha)((\Gamma(b+\alpha) / \Gamma(b+1))-$ $\Gamma(\alpha))$.

\section{Antiperiodic Boundary Value Problem}

In this section, we consider the following antiperiodic boundary value problem:

$$
\begin{aligned}
& \Delta_{C}^{\alpha} x(t)=f(t+\alpha-1, x(t+\alpha-1)), \quad t \in[0, b]_{\mathbb{N}_{0}}, 1<\alpha<2, \\
& x(\alpha-1)+x(b+\alpha)=0, \quad \Delta x(\alpha-1)+\Delta x(b+\alpha-1)=0,
\end{aligned}
$$

where $\Delta$ is a forward difference operator.

Let $B$ be the set of all real sequences $x=\{x(t)\}_{t=\alpha-1}^{b+\alpha}$ with norm $\|x\|=\sup _{t \in[\alpha-1, b+\alpha]_{N_{\alpha-1}}}|x(t)|$. Then, $B$ is a Banach space.

Lemma 6. A solution $x: t \in[\alpha-1, b+\alpha]_{\mathbb{N}_{\alpha-1}} \times R \rightarrow R$ is a solution for antiperiodic boundary value problem

$$
\begin{gathered}
\Delta_{C}^{\alpha} x(t)=f(t+\alpha-1), \quad t \in[0, b]_{\mathbb{N}_{0}}, 1<\alpha<2, \\
x(\alpha-1)+x(b+\alpha)=0, \quad \Delta x(\alpha-1)+\Delta x(b+\alpha-1)=0,
\end{gathered}
$$


if and only if $x(t)$ is a solution of the the following fractional Taylor's difference formula:

$$
\begin{array}{rl}
x(t)= & \frac{1}{\Gamma(\alpha)} \sum_{s=0}^{t-\alpha}(t-s-1)^{(\alpha-1)} f(s+\alpha-1) \\
& -\frac{1}{2 \Gamma(\alpha)} \sum_{s=0}^{b}(b+\alpha-s-1)^{(\alpha-1)} f(s+\alpha-1) \\
& +\frac{b+2 \alpha-1-t}{2 \Gamma(\alpha-1)} \sum_{s=0}^{b-1}(b+\alpha-s-2)^{(\alpha-2)} f(s+\alpha-1), \\
t & t \in[\alpha-1, b+\alpha]_{\mathbb{N}_{\alpha-1}} .
\end{array}
$$

Proof. Suppose that $x(t)$ defined on $[\alpha-1, b+\alpha]_{\mathbb{N}_{\alpha-1}}$ is a solution of (10). Using Lemma 3, for some constants $c_{0}, c_{1} \in$ $R$, we have

$$
\begin{aligned}
& x(t)= \Delta^{-\alpha} f(t+\alpha-1)-c_{0}-c_{1} t \\
&=\frac{1}{\Gamma(\alpha)} \sum_{s=0}^{t-\alpha}(t-s-1)^{(\alpha-1)} f(s+\alpha-1)-c_{0}-c_{1} t, \\
& t \in[\alpha-1, b+\alpha]_{\mathbb{N}_{\alpha-1}} .
\end{aligned}
$$

Then, we obtain [3]

$$
\begin{array}{r}
\Delta x(t)=\frac{1}{\Gamma(\alpha-1)} \sum_{s=0}^{t-\alpha}(t-s-1)^{(\alpha-2)} f(s+\alpha-1)-c_{1}, \\
t \in[\alpha-1, b+\alpha-1]_{\mathbb{N}_{\alpha-1}} .
\end{array}
$$

In view of $x(\alpha-1)+x(b+\alpha)=0$ and $\Delta x(\alpha-1)+\Delta x(b+$ $\alpha-1)=0$, we have

$$
\begin{gathered}
\frac{1}{\Gamma(\alpha)} \sum_{s=0}^{b}(b+\alpha-s-1)^{(\alpha-1)} f(s+\alpha-1)-2 c_{0}-c_{1}(b+2 \alpha-1)=0, \\
\frac{1}{\Gamma(\alpha-1)} \sum_{s=0}^{b-1}(b+\alpha-s-2)^{(\alpha-2)} f(s+\alpha-1)-2 c_{1}=0 .
\end{gathered}
$$

Then,

$$
\begin{aligned}
c_{0}= & \frac{1}{2 \Gamma(\alpha)} \sum_{s=0}^{b}(b+\alpha-s-1)^{(\alpha-1)} f(s+\alpha-1) \\
& -\frac{b+2 \alpha-1}{2 \Gamma(\alpha-1)} \sum_{s=0}^{b-1}(b+\alpha-s-2)^{(\alpha-2)} f(s+\alpha-1), \\
c_{1}= & \frac{1}{2 \Gamma(\alpha-1)} \sum_{s=0}^{b-1}(b+\alpha-s-2)^{(\alpha-2)} f(s+\alpha-1) .
\end{aligned}
$$

Substituting the values of $c_{0}$ and $c_{1}$ into (12), we obtain (11).

Conversely, if $x(t)$ is a solution of (11), by a direct computation, it follows that the solution given by (11) satisfies (10). The proof is completed.
The following fixed-point theorems are needed to prove the existence and uniqueness of solutions for the BVP (9).

Lemma 7 (see [15] (Banach contraction mapping principle)). A contraction mapping on a complete metric space has exactly one fixed point.

Lemma 8 (see [16] (Brower fixed-point theorem)). Let $F$ : $C \in R^{n} \rightarrow C \in R^{n}$ be a continuous mapping, where $C$ is a nonempty, bounded, close, and convex set. Then, $F$ has a fixed point.

Define the operator

$(T x)(t)$

$$
\begin{aligned}
= & \frac{1}{\Gamma(\alpha)} \sum_{s=0}^{t-\alpha}(t-s-1)^{(\alpha-1)} f(s+\alpha-1, x(s+\alpha-1)) \\
& -\frac{1}{2 \Gamma(\alpha)} \sum_{s=0}^{b}(b+\alpha-s-1)^{(\alpha-1)} f(s+\alpha-1, x(s+\alpha-1)) \\
& +\frac{b+2 \alpha-1-t}{2 \Gamma(\alpha-1)} \sum_{s=0}^{b-1}(b+\alpha-s-2)^{(\alpha-2)} \\
& \times f(s+\alpha-1, x(s+\alpha-1)), \quad t \in[\alpha-1, b+\alpha]_{\mathbb{N}_{\alpha-1}} .
\end{aligned}
$$

Obviously, $x(t)$ is a solution of (9) if it is a fixed point of the operator $T$.

Theorem 9. Assume that.

$\left(H_{1}\right)$ There exists a constant $L>0$ such that $\mid f(t, x)-$ $f(t, y)|\leq L| x-y \mid$ for each $t \in[\alpha-1, b+\alpha]_{\mathbb{N}_{\alpha-1}}$ and all $x, y \in B$.

Then, the BVP (9) has a unique solution on B provided that

$$
L<\frac{3 \Gamma(b+\alpha+1)}{2 \Gamma(\alpha+1) \Gamma(b+1)}+\frac{b+\alpha}{2 \alpha \Gamma(\alpha-1)}\left(\frac{\Gamma(b+\alpha)}{\Gamma(b+1)}-\Gamma(\alpha)\right)
$$

Proof. Let $x, y \in B$; then for each $t \in[\alpha-1, b+\alpha]_{\mathbb{N}_{\alpha-1}}$, we have

$$
\begin{aligned}
& |(T x)(t)-(T y)(t)| \\
& \leq \frac{1}{\Gamma(\alpha)} \sum_{s=0}^{t-\alpha}(t-s-1)^{(\alpha-1)} \\
& \quad \times \mid f(s+\alpha-1, x(s+\alpha-1)) \\
& \quad-f(s+\alpha-1, y(s+\alpha-1)) \mid \\
& +\frac{1}{2 \Gamma(\alpha)} \sum_{s=0}^{b}(b+\alpha-s-1)^{(\alpha-1)}
\end{aligned}
$$




$$
\begin{aligned}
& \times \mid f(s+\alpha-1, x(s+\alpha-1)) \\
& -f(s+\alpha-1, y(s+\alpha-1)) \mid \\
& +\frac{|b+2 \alpha-1-t|}{2 \Gamma(\alpha-1)} \sum_{s=0}^{b-1}(b+\alpha-s-2)^{(\alpha-2)} \\
& \times \mid f(s+\alpha-1, x(s+\alpha-1)) \\
& -f(s+\alpha-1, y(s+\alpha-1)) \mid \\
& \leq \frac{L}{\Gamma(\alpha)} \sum_{s=0}^{t-\alpha}(t-s-1)^{(\alpha-1)} \\
& \times|x(s+\alpha-1)-y(s+\alpha-1)| \\
& +\frac{L}{2 \Gamma(\alpha)} \sum_{s=0}^{b}(b+\alpha-s-1)^{(\alpha-1)} \\
& \times|x(s+\alpha-1)-y(s+\alpha-1)| \\
& +\frac{L|b+2 \alpha-1-t|}{2 \Gamma(\alpha-1)} \sum_{s=0}^{b-1}(b+\alpha-s-2)^{(\alpha-2)} \\
& \times|x(s+\alpha-1)-y(s+\alpha-1)| \\
& \leq \frac{L\|x-y\|}{\Gamma(\alpha)} \sum_{s=0}^{t-\alpha}(t-s-1)^{(\alpha-1)}+\frac{L\|x-y\|}{2 \Gamma(\alpha)} \\
& \times \sum_{s=0}^{b}(b+\alpha-s-1)^{(\alpha-1)} \\
& +\frac{|b+2 \alpha-1-t| \cdot L\|x-y\|}{2 \Gamma(\alpha-1)} \sum_{s=0}^{b-1}(b+\alpha-s-2)^{(\alpha-2)} \\
& \leq \frac{L\|x-y\|}{2 \Gamma(\alpha)} \cdot \frac{\Gamma(t+1)}{\alpha \Gamma(t-\alpha+1)} \\
& +\frac{L\|x-y\|}{2 \Gamma(\alpha)} \cdot \frac{\Gamma(b+\alpha+1)}{\alpha \Gamma(b+1)} \\
& +\frac{L(b+\alpha)\|x-y\|}{2 \Gamma(\alpha-1)} \cdot \frac{1}{\alpha}\left(\frac{\Gamma(b+\alpha)}{\Gamma(b+1)}-\Gamma(\alpha)\right) \\
& \leq\left[\frac{3 \Gamma(b+\alpha+1)}{2 \Gamma(\alpha+1) \Gamma(b+1)}\right. \\
& \left.+\frac{b+\alpha}{2 \alpha \Gamma(\alpha-1)}\left(\frac{\Gamma(b+\alpha)}{\Gamma(b+1)}-\Gamma(\alpha)\right)\right] L\|x-y\| .
\end{aligned}
$$

According to (17), we obtain

$$
|(T x)(t)-(T y)(t)|<\|x-y\| .
$$

Then,

$$
\|T x-T y\| \leq\|x-y\|
$$

which implies that $T$ is a contraction. Therefore, the Banach fixed-point theorem (Lemma 7) guarantees that $T$ has a unique fixed point which is a unique solution of the BVP (9). This completes the proof.

Theorem 10. Assume that.

$\left(H_{2}\right)$ There exists a bounded function $K:[\alpha-1, b+\alpha]_{\mathbb{N}_{\alpha-1}}$ $\rightarrow R$ such that $|f(t, x)| \leq K(t)|x|$ for all $x \in B$.

Then, the BVP (9) has at least a solution on $B$ provided that

$$
K^{*}<\frac{3 \Gamma(b+\alpha+1)}{2 \Gamma(\alpha+1) \Gamma(b+1)}+\frac{b+\alpha}{2 \alpha \Gamma(\alpha-1)}\left(\frac{\Gamma(b+\alpha)}{\Gamma(b+1)}-\Gamma(\alpha)\right),
$$

where $K^{*}=\max \left\{K(t): t \in[\alpha-1, b+\alpha]_{\mathbb{N}_{\alpha-1}}\right\}$.

Proof. Let $M>0$; define the set

$$
S=\left\{x(t) \mid[\alpha-1, b+\alpha]_{\mathbb{N}_{\alpha-1}} \longrightarrow R,\|x\| \leq M\right\} .
$$

To prove this theorem, we only need to show that $T$ maps $S$ in $S$.

For $x(t) \in S$, we have

$|(T x)(t)|$

$$
\begin{aligned}
\leq & \frac{K(t)}{\Gamma(\alpha)} \sum_{s=0}^{t-\alpha}(t-s-1)^{(\alpha-1)}|x(s+\alpha-1)| \\
& +\frac{K(t)}{2 \Gamma(\alpha)} \sum_{s=0}^{b}(b+\alpha-s-1)^{(\alpha-1)}|x(s+\alpha-1)| \\
& +\frac{(b+2 \alpha-1-t) K(t)}{2 \Gamma(\alpha-1)} \\
& \times \sum_{s=0}^{b-1}(b+\alpha-s-2)^{(\alpha-2)}|x(s+\alpha-1)| \\
\leq & \frac{K(t)\|x\|^{t-\alpha}}{\Gamma(\alpha)} \sum_{s=0}(t-s-1)^{(\alpha-1)} \\
& +\frac{K(t)\|x\|}{2 \Gamma(\alpha)} \sum_{s=0}^{b}(b+\alpha-s-1)^{(\alpha-1)} \\
& +\frac{(b+2 \alpha-1-t) K(t)\|x\|}{2 \Gamma(\alpha-1)} \sum_{s=0}^{b-1}(b+\alpha-s-2)^{(\alpha-2)} \\
\leq & \frac{K(t)\|x\|}{2 \Gamma(\alpha)} \cdot \frac{\Gamma(t+1)}{\alpha \Gamma(t-\alpha+1)}+\frac{K(t)\|x\|}{2 \Gamma(\alpha)} \cdot \frac{\Gamma(b+\alpha+1)}{\alpha \Gamma(b+1)} \\
& +\frac{K(t)(b+\alpha)\|x\|}{2 \Gamma(\alpha-1)} \cdot \frac{1}{\alpha}\left(\frac{\Gamma(b+\alpha)}{\Gamma(b+1)}-\Gamma(\alpha)\right)
\end{aligned}
$$




$$
\begin{aligned}
\leq & {\left[\frac{3 \Gamma(b+\alpha+1)}{2 \Gamma(\alpha+1) \Gamma(b+1)}\right.} \\
& \left.+\frac{b+\alpha}{2 \alpha \Gamma(\alpha-1)}\left(\frac{\Gamma(b+\alpha)}{\Gamma(b+1)}-\Gamma(\alpha)\right)\right] K(t)\|x\| \\
\leq & {\left[\frac{3 \Gamma(b+\alpha+1)}{2 \Gamma(\alpha+1) \Gamma(b+1)}\right.} \\
& \left.+\frac{b+\alpha}{2 \alpha \Gamma(\alpha-1)}\left(\frac{\Gamma(b+\alpha)}{\Gamma(b+1)}-\Gamma(\alpha)\right)\right] K^{*} M .
\end{aligned}
$$

From (21), we have $|(T x)(t)|<M$; then, $\|T x\| \leq M$ which implies that $T$ maps $S$ in $S$. $T$ has at least a fixed point which is a solution of the BVP (9) according to Brower fixed-point theorem (Lemma 8). This completes the proof.

\section{The Ulam Stability}

Similar to the definitions of the Ulam stability for fractional differential equation [14], we introduce four types of the Ulam stability definitions for fractional difference equation.

Consider (1) and the following inequalities:

$$
\begin{array}{r}
\left|\Delta_{C}^{\alpha} y(t)-f(t+\alpha-1, y(t+\alpha-1))\right| \leq \varepsilon, \quad t \in[0, b]_{\mathbb{N}_{0}}, \\
\left|\Delta_{C}^{\alpha} y(t)-f(t+\alpha-1, y(t+\alpha-1))\right| \leq \varepsilon \varphi(t+\alpha-1) \\
t \in[0, b]_{\mathbb{N}_{0}} .
\end{array}
$$

Definition 11. Equation (1) is the Ulam-Hyers stable if there exists a real number $c_{f}>0$ such that for each $\varepsilon>0$ and for each solution $y \in B$ of inequality (24), there exists a solution $x \in B$ of (1) with

$$
|y(t)-x(t)| \leq c_{f} \mathcal{E}, \quad t \in[\alpha-1, b+\alpha]_{\mathbb{N}_{\alpha-1}} .
$$

Equation (1) is the generalized Ulam-Hyers stable if we substitute the function $\theta_{f}(\varepsilon)$ for the constant $c_{f} \mathcal{E}$ on inequality (26), where $\theta_{f}(\varepsilon) \in C\left(R^{+}, R^{+}\right)$and $\theta_{f}(0)=0$.

Definition 12. Equation (1) is the Ulam-Hyers-Rassias stable with respect to $\varphi$ if there exists a real number $c_{f, \varphi}>0$ such that for each $\varepsilon>0$ and for each solution $y \in B$ of inequality (25), there exists a solution $x \in B$ of (1) with

$$
|y(t)-x(t)| \leq c_{f, \varphi} \varepsilon \varphi(t), \quad t \in[\alpha-1, b+\alpha]_{\mathbb{N}_{\alpha-1}} .
$$

Equation (1) is the generalized Ulam-Hyers-Rassias stable if we substitute the function $\varphi(t)$ for the function $\varepsilon \varphi(t)$ on inequalities (25) and (27).

Remark 13. If $\varphi$ is a constant function in Definition 12, we say that the integral equation (25) has also the Hyers-Ulam stability.

Remark 14. A function $y \in B$ is a solution of inequality (24) if and only if there exists a function $g:[\alpha-1, b+\alpha-1]_{\mathbb{N}_{\alpha-1}} \rightarrow$ $R$ such that (i) $|g(t+\alpha-1)| \leq \varepsilon, t \in[0, b]_{\mathbb{N}_{0}}$,

(ii) $\Delta_{C}^{\alpha} y(t)=f(t+\alpha-1, y(t+\alpha-1))+g(t+\alpha-1), t \in$ $[0, b]_{\mathbb{N}_{0}}$,

similar remark for inequality (25).

Theorem 15. Assume that $\left(H_{1}\right)$ holds. Let $y \in B$ be a solution of inequality (24) and let $x \in B$ be a solution of the following boundary value problem:

$$
\begin{gathered}
\Delta_{C}^{\alpha} x(t)=f(t+\alpha-1, x(t+\alpha-1)), \quad t \in[0, b]_{\mathbb{N}_{0}}, 1<\alpha<2, \\
x(\alpha-1)=y(\alpha-1), \quad x(b+\alpha)=y(b+\alpha) .
\end{gathered}
$$

Then, (1) is the Ulam-Hyers stable provided that

$$
L<\frac{\Gamma(\alpha+1) \Gamma(b+1)}{2 \Gamma(b+\alpha+1)} .
$$

Proof. By Lemma 3, the solution of the BVP (28) is given by

$$
\begin{aligned}
x(t)= & y(\alpha-1)+\frac{t}{b+\alpha}(y(b+\alpha)-y(\alpha-1)) \\
& -\frac{t}{(b+\alpha) \Gamma(\alpha)} \sum_{s=0}^{b}(b+\alpha-s-1)^{(\alpha-1)} \\
& \times f(s+\alpha-1, x(s+\alpha-1))+\frac{1}{\Gamma(\alpha)} \sum_{s=0}^{t-\alpha}(t-s-1)^{(\alpha-1)} \\
& \times f(s+\alpha-1, x(s+\alpha-1)), \quad t \in[\alpha-1, b+\alpha]_{\mathbb{N}_{\alpha-1}} .
\end{aligned}
$$

From inequality (24), for $t \in[\alpha-1, b+\alpha]_{\mathbb{N}_{\alpha-1}}$, it follows that

$$
\begin{aligned}
& \mid y(t)-y(\alpha-1)-\frac{t}{b+\alpha}(y(b+\alpha)-y(\alpha-1)) \\
& +\frac{t}{(b+\alpha) \Gamma(\alpha)} \sum_{s=0}^{b}(b+\alpha-s-1)^{(\alpha-1)} \\
& \times f(s+\alpha-1, y(s+\alpha-1)) \\
& -\frac{1}{\Gamma(\alpha)} \sum_{s=0}^{t-\alpha}(t-s-1)^{(\alpha-1)} f(s+\alpha-1, y(s+\alpha-1)) \mid \\
& \quad \leq \frac{\varepsilon}{\Gamma(\alpha)} \sum_{s=0}^{t-\alpha}(t-s-1)^{(\alpha-1)} \\
& \quad=\frac{\varepsilon}{\Gamma(\alpha)} \cdot \frac{\Gamma(t+1)}{\alpha \Gamma(t-\alpha+1)} \\
& \quad \leq \frac{\Gamma(b+\alpha+1)}{\Gamma(\alpha+1) \Gamma(b+1)} \varepsilon .
\end{aligned}
$$


Combining (30) and (31), for $t \in[\alpha-1, b+\alpha]_{\mathbb{N}_{\alpha-1}}$, we have

$$
\begin{aligned}
& |y(t)-x(t)| \\
& \leq \mid y(t)-y(\alpha-1)-\frac{t}{b+\alpha}(y(b+\alpha)-y(\alpha-1)) \\
& +\frac{t}{(b+\alpha) \Gamma(\alpha)} \\
& \times \sum_{s=0}^{b}(b+\alpha-s-1)^{(\alpha-1)} f(s+\alpha-1, x(s+\alpha-1)) \\
& -\frac{1}{\Gamma(\alpha)} \sum_{s=0}^{t-\alpha}(t-s-1)^{(\alpha-1)} \\
& \times f(s+\alpha-1, x(s+\alpha-1)) \\
& \leq \mid y(t)-y(\alpha-1)-\frac{t}{b+\alpha}(y(b+\alpha)-y(\alpha-1)) \\
& +\frac{t}{(b+\alpha) \Gamma(\alpha)} \\
& \times \sum_{s=0}^{b}(b+\alpha-s-1)^{(\alpha-1)} f(s+\alpha-1, y(s+\alpha-1)) \\
& -\frac{1}{\Gamma(\alpha)} \sum_{s=0}^{t-\alpha}(t-s-1)^{(\alpha-1)} \\
& \times f(s+\alpha-1, y(s+\alpha-1)) \\
& +\frac{t}{(b+\alpha) \Gamma(\alpha)} \sum_{s=0}^{b}(b+\alpha-s-1)^{(\alpha-1)} \\
& \times \mid f(s+\alpha-1, y(s+\alpha-1)) \\
& -f(s+\alpha-1, x(s+\alpha-1)) \mid \\
& +\frac{1}{\Gamma(\alpha)} \sum_{s=0}^{t-\alpha}(t-s-1)^{(\alpha-1)} \\
& \times \mid f(s+\alpha-1, y(s+\alpha-1)) \\
& -f(s+\alpha-1, x(s+\alpha-1)) \mid \\
& \leq \frac{\Gamma(b+\alpha+1)}{\Gamma(\alpha+1) \Gamma(b+1)} \varepsilon+\frac{m_{f} t}{(b+\alpha) \Gamma(\alpha)} \\
& \times \sum_{s=0}^{b}(b+\alpha-s-1)^{(\alpha-1)} \\
& \times|y(s+\alpha-1)-x(s+\alpha-1)| \\
& +\frac{m_{f}}{\Gamma(\alpha)} \sum_{s=0}^{t-\alpha}(t-s-1)^{(\alpha-1)} \\
& \times|y(s+\alpha-1)-x(s+\alpha-1)|
\end{aligned}
$$

$$
\begin{aligned}
& \leq \frac{\Gamma(b+\alpha+1)}{\Gamma(\alpha+1) \Gamma(b+1)} \varepsilon+\frac{m_{f} t\|y-x\|}{(b+\alpha) \Gamma(\alpha)} \\
& \times \sum_{s=0}^{b}(b+\alpha-s-1)^{(\alpha-1)} \\
& +\frac{m_{f}\|y-x\|}{\Gamma(\alpha)} \sum_{s=0}^{t-\alpha}(t-s-1)^{(\alpha-1)} \\
& =\frac{\Gamma(b+\alpha+1)}{\Gamma(\alpha+1) \Gamma(b+1)} \varepsilon+\frac{m_{f} t}{(b+\alpha) \Gamma(\alpha)} \\
& \frac{1}{\alpha} \frac{\Gamma(b+\alpha+1)}{\Gamma(b+1)}\|y-x\| \\
& +\frac{m_{f}}{\Gamma(\alpha)} \cdot \frac{\Gamma(t+1)}{\alpha \Gamma(t-\alpha+1)}\|y-x\| \\
& \leq \frac{\Gamma(b+\alpha+1)}{\Gamma(\alpha+1) \Gamma(b+1)} \varepsilon+\frac{m_{f} t}{(b+\alpha) \Gamma(\alpha)} \\
& \cdot \frac{1}{\alpha} \frac{\Gamma(b+\alpha+1)}{\Gamma(b+1)}\|y-x\| \\
& +\frac{m_{f}}{\Gamma(\alpha)} \cdot \frac{\Gamma(t+1)}{\alpha \Gamma(t-\alpha+1)}\|y-x\| \\
& \leq \frac{\Gamma(b+\alpha+1)}{\Gamma(\alpha+1) \Gamma(b+1)} \varepsilon+\frac{2 m_{f} \Gamma(b+\alpha+1)}{\Gamma(\alpha+1) \Gamma(b+1)} \\
& \times\|y-x\| \text {. }
\end{aligned}
$$

Then,

$$
\begin{aligned}
\|y-x\| \leq & \frac{\Gamma(b+\alpha+1)}{\Gamma(\alpha+1) \Gamma(b+1)} \varepsilon \\
& +\frac{2 L \Gamma(b+\alpha+1)}{\Gamma(\alpha+1) \Gamma(b+1)}\|y-x\| .
\end{aligned}
$$

Applying (29) to the previous inequality yields that

$$
\|y-x\| \leq \frac{\Gamma(b+\alpha+1)}{\Gamma(\alpha+1) \Gamma(b+1)-2 L \Gamma(b+\alpha+1)} \varepsilon ;
$$

where $\Gamma(b+\alpha+1) /(\Gamma(\alpha+1) \Gamma(b+1)-2 L \Gamma(b+\alpha+1))>0$, thus; (1) is the Ulam-Hyers stable.

Theorem 16. Assume that $\left(H_{1}\right)$ and the following condition hold.

$\left(H_{3}\right)$ Let $\varphi:[\alpha-1, b+\alpha]_{\mathbb{N}_{\alpha-1}} \rightarrow R^{+}$be an increasing function. There exists a constant $\lambda^{\alpha-1}>0$ such that

$$
\begin{array}{r}
\frac{1}{\Gamma(\alpha)} \sum_{s=0}^{t-\alpha}(t-s-1)^{(\alpha-1)} \varphi(s+\alpha-1) \leq \lambda \varepsilon \varphi(t+\alpha-1) \\
t \in[0, b]_{\mathbb{N}_{0}} .
\end{array}
$$

Let $y \in B$ be a solution of inequality (25) and let $x \in B$ be a solution of the following boundary value problem (28). Then, (1) is the Ulam-Hyers-Rassias stable provided that (29) holds. 
The proof of Theorem 16 is similar to that of Theorem 15, and we omit it.

\section{Examples}

As the applications of our main results, we consider the following examples.

Example 1. Consider the fractional difference BVP

$$
\begin{gathered}
\Delta_{C}^{1.5} x(t)=\lambda x(t+0.5), \quad t \in[0,10]_{\mathbb{N}_{0}}, \\
x(0.5)+x(11.5)=0, \quad \Delta x(0.5)+\Delta x(10.5)=0,
\end{gathered}
$$

where $f(t, x)=\lambda x(t)$ for $t \in[0.5,11.5]_{\mathbb{N}_{0.5}}$ and conditions $\left(H_{1}\right)$ and $\left(H_{2}\right)$ are satisfied. Since

$$
\begin{aligned}
& \frac{3 \Gamma(b+\alpha+1)}{2 \Gamma(\alpha+1) \Gamma(b+1)}+\frac{b+\alpha}{2 \alpha \Gamma(\alpha-1)}\left(\frac{\Gamma(b+\alpha)}{\Gamma(b+1)}-\Gamma(\alpha)\right) \\
& \approx 47.7268
\end{aligned}
$$

inequalities (17) and (21) are satisfied if $\lambda<47.7268$. According to Theorem 9, the BVP (36) has a unique solution. At the same time, the BVP (36) has at least a solution by Theorem 10.

Example 2. Consider the fractional difference equation

$$
\Delta_{C}^{1.5} x(t)=\lambda x(t+0.5), \quad t \in[0,10]_{\mathbb{N}_{0}},
$$

with the boundary conditions

$$
x(0.5)=y(0.5), \quad x(11.5)=y(11.5) .
$$

Since

$$
\frac{\Gamma(\alpha+1) \Gamma(b+1)}{2 \Gamma(b+\alpha+1)} \approx 0.0176,
$$

if $\lambda<0.0176$ and the inequality

$$
\left|\Delta_{C}^{1.5} y(t)-f(t+0.5, y(t+0.5))\right| \leq \varepsilon, \quad \text { for } t \in[0,10]_{\mathbb{N}_{0}},
$$

hold, then (38) is the Ulam-Hyers stable by Theorem 15.

\section{Acknowledgments}

This work was supported in part by the Natural Science Foundation of China (10971173), the National Natural Science Foundation of Hunan Province under Grant 13JJ3120, and the Construct Program of the Key Discipline in Hunan Province.

\section{References}

[1] G. A. Anastassiou, "Nabla discrete fractional calculus and nabla inequalities," Mathematical and Computer Modelling, vol. 51, no. 5-6, pp. 562-571, 2010.

[2] T. Abdeljawad, "On Riemann and Caputo fractional differences," Computers \& Mathematics with Applications, vol. 62, no. 3, pp. 1602-1611, 2011.
[3] F. M. Atici and P. W. Eloe, "Initial value problems in discrete fractional calculus," Proceedings of the American Mathematical Society, vol. 137, no. 3, pp. 981-989, 2009.

[4] F. M. Atici and P. W. Eloe, "A transform method in discrete fractional calculus," International Journal of Difference Equations, vol. 2, no. 2, pp. 165-176, 2007.

[5] F. M. Atıcı and P. W. Eloe, "Discrete fractional calculus with the nabla operator," Electronic Journal of Qualitative Theory of Differential Equations, vol. 3, pp. 1-12, 2009.

[6] F. M. Atıcı and S. Şengül, "Modeling with fractional difference equations," Journal of Mathematical Analysis and Applications, vol. 369, no. 1, pp. 1-9, 2010.

[7] R. A. C. Ferreira, "A discrete fractional Gronwall inequality," Proceedings of the American Mathematical Society, vol. 140, no. 5, pp. 1605-1612, 2012.

[8] F. Chen, X. Luo, and Y. Zhou, "Existence results for nonlinear fractional difference equation," Advances in Difference Equations, vol. 2011, Article ID 713201, 12 pages, 2011.

[9] F. Chen, "Fixed points and asymptotic stability of nonlinear fractional difference equations," Electronic Journal of Qualitative Theory of Differential Equations, vol. 39, pp. 1-18, 2011.

[10] F. Chen and Z. Liu, "Asymptotic stability results for nonlinear fractional difference equations," Journal of Applied Mathematics, vol. 2012, Article ID 879657, 14 pages, 2012.

[11] C. S. Goodrich, "Existence of a positive solution to a system of discrete fractional boundary value problems," Applied Mathematics and Computation, vol. 217, no. 9, pp. 4740-4753, 2011.

[12] C. S. Goodrich, "On discrete sequential fractional boundary value problems," Journal of Mathematical Analysis and Applications, vol. 385, no. 1, pp. 111-124, 2012.

[13] W. Lv, "Existence of solutions for discrete fractional boundary value problems with a $p$-Laplacian operator," Advances in Difference Equations, vol. 2012, article 163, 2012.

[14] J. Wang, L. Lv, and Y. Zhou, "Ulam stability and data dependence for fractional differential equations with Caputo derivative," Electronic Journal of Qualitative Theory of Differential Equations, vol. 63, pp. 1-10, 2011.

[15] A. N. Kolmogorov and S. V. Fomin, Elements of Function Theory and Functional Analysis, Nauka, Moscow, Russia, 1981.

[16] R. P. Agarwal, M. Meehan, and D. O'Regan, Fixed Point Theory and Applications, vol. 141 of Cambridge Tracts in Mathematics, Cambridge University Press, Cambridge, UK, 2001. 


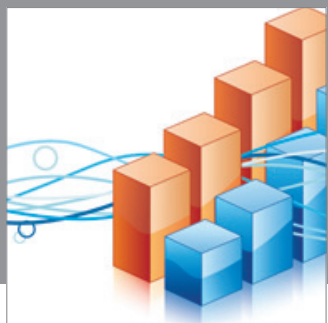

Advances in

Operations Research

mansans

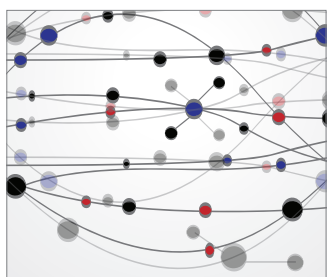

The Scientific World Journal
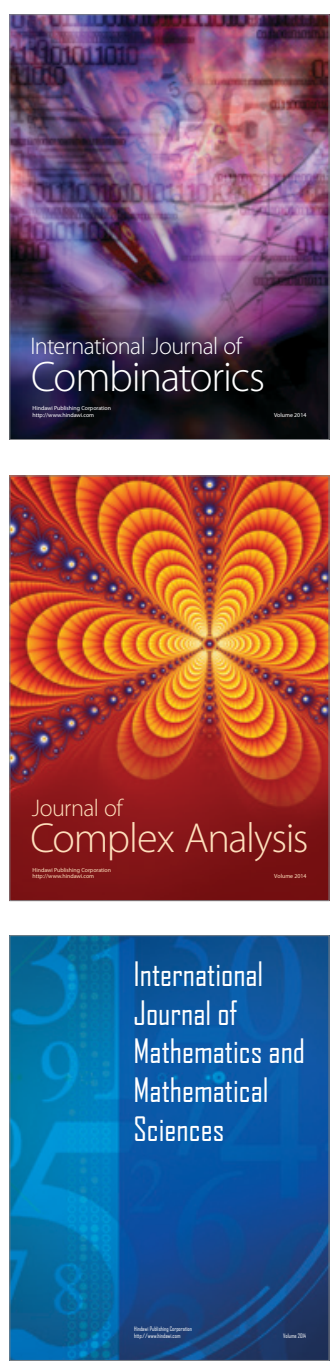
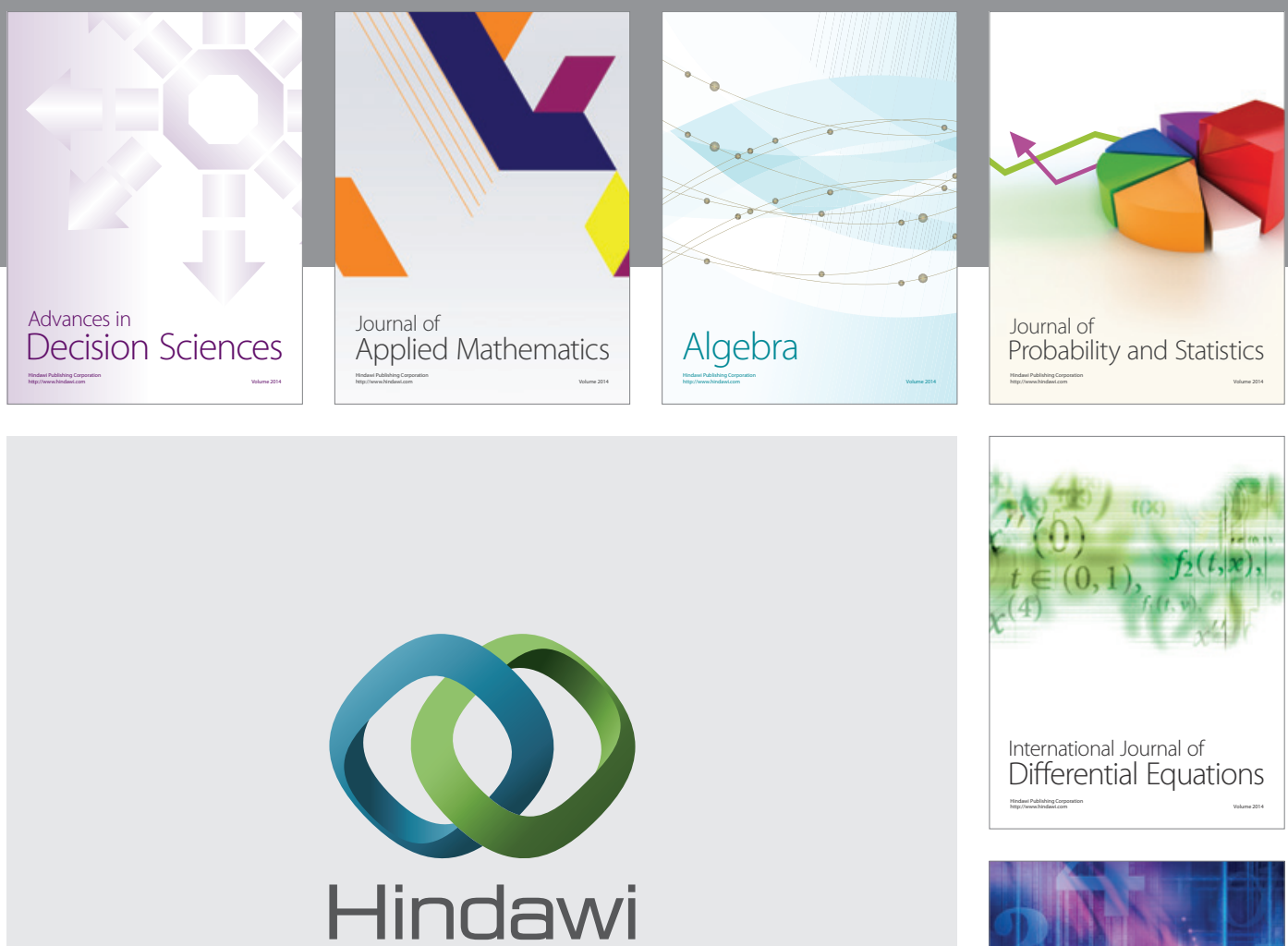

Submit your manuscripts at http://www.hindawi.com
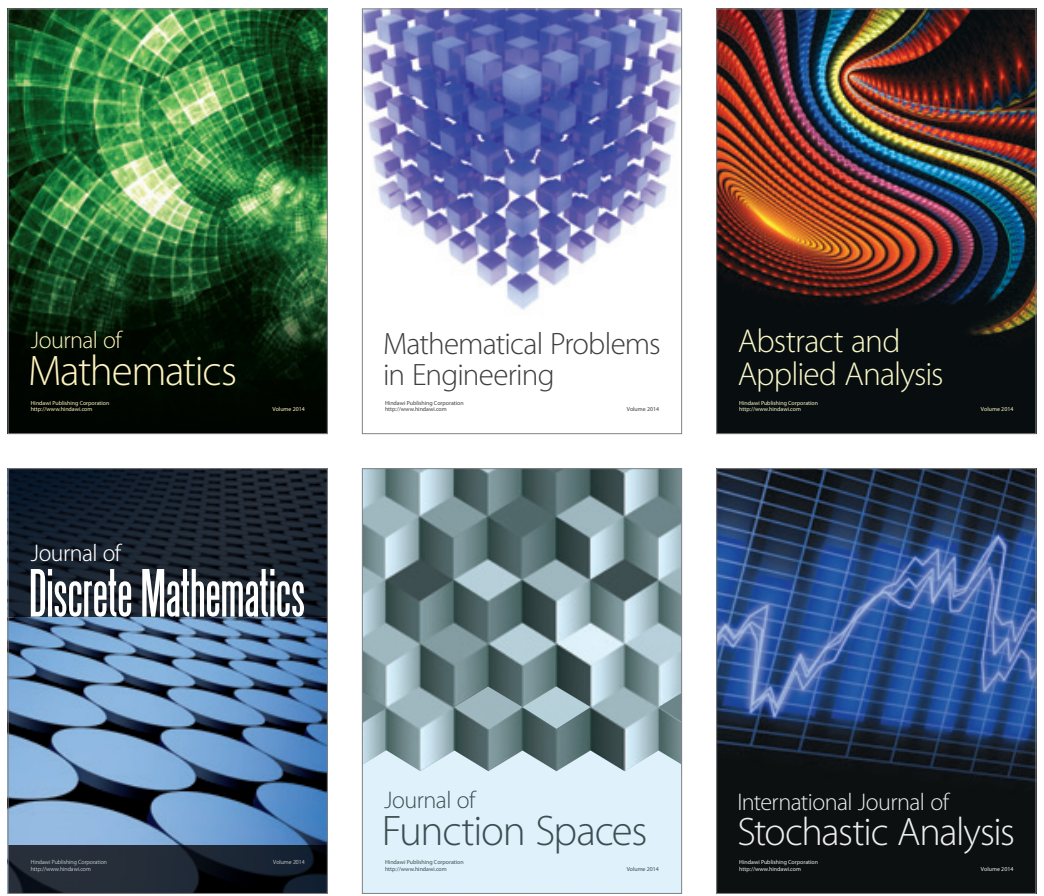

Journal of

Function Spaces

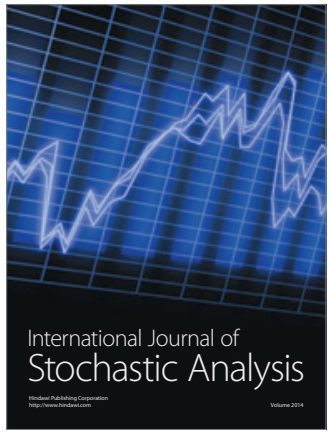

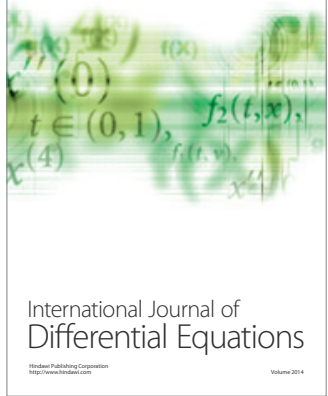
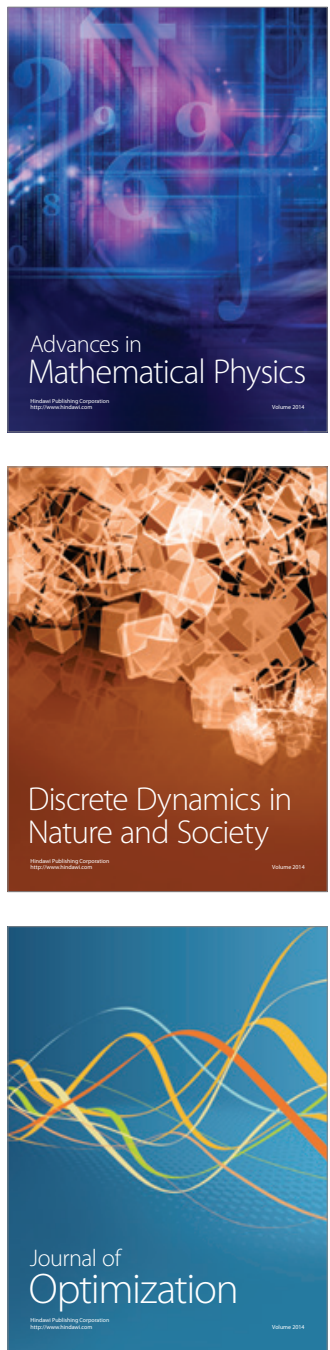